\title{
Magnetization rotation or generation of incoherent spin waves? Suggestions for a spin-transfer effect experiment.
}

\author{
Ya.B. Bazaliy a,1, B.A. Jones ${ }^{\text {b }}$, \\ a Argonne National Laboratory, MSD, 9700 S. Cass Ave, Argonne, IL 60439, USA \\ b IBM Almaden Research Center, 650 Harry Road, San Jose, CA 95120, USA.
}

\begin{abstract}
"Spin-transfer" torque is created when electric current is passed through metallic ferromagnets and may have interesting applications in spintronics. So far it was experimentally studied in "collinear" geometries, where it is difficult to predict whether magnetization will coherently rotate or spin-waves will be generated. Here we propose an easy modification of existing experiment in which the spin-polarization of incoming current will no longer be collinear with magnetization and recalculate the switching behavior of the device. We expect that a better agreement with the magnetization rotation theory will be achieved. That can be an important step in reconciling alternative points of view on the effect of spin-transfer torque.
\end{abstract}

Key words: spintronics; spin-transfer effect; current induced switching

\section{Effects of spin-transfer torque}

Electric current flowing from one metallic ferromagnet to another induces an interaction between them $[1,2]$. This interaction is qualitatively different from the one observed in equilibrium and creates a so-called "spin-transfer" torque - a subject of recent interest in the field of spintronics with large promise for future applications. Spin-transfer was studied in several experiments, see e.g. $[3,4]$.

The result of spin-transfer torque action depends on the most easily excited magnetic mode coupled to the spin-polarized current. Currently there are two views on the nature of this mode. In one approach $[1,5]$ it is assumed that a coherent rotation of magnetization is induced (in [6] a more general case space-dependent but still coherent spin waves were studied) and in the other $[2,7]$ - that incoherent spin waves are generated. Since spin-transfer torque is generated in a thin layer near the N/F bundaries, it generally is coupled to both modes. The question of the relative coupling strengths

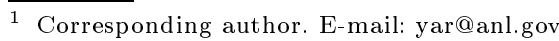

Preprint submitted to LT23 Proceedings
(A)

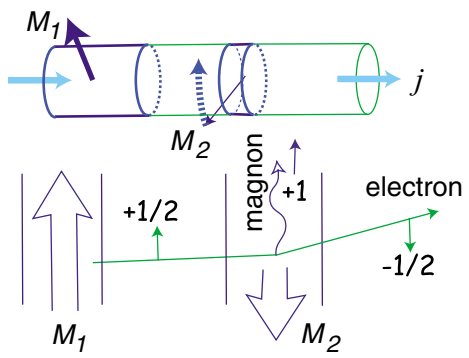

Fig. 1. Possible outcomes of the spin-transfer torque generated by a spin-polarized current coming from the magnet $M_{1}$. A: coherent rotation of the $M_{2}$ magnetization. B: creation of spin waves in the second magnetic layer

is difficult and is not worked out yet. However there is a parameter which can favor one type of excitations over the other, and this is the initial angle between the magnetizations of the ferromagnets. Expression for the spin-transfer torque in the coherent rotation mode [1] vanishes for the case of collinear magnetizations. At the same time, processes in which individual electrons create incoherent spin waves are still allowed (see Fig.1). This is why they were considered as most important in 
$[2,7]$.

In that sense collinear case is the least advantageous arrangement for the coherent rotation hypothesis to hold. In this geometry coherent rotation can only be initiated if a fluctuation of average magnetization direction would happen. Then the theory predicts that under certain conditions spin transfer torque will cause the growth of such fluctuation.

So far the effect of spin-transfer torque was studied in the collinear setup [3]. In [5] exact theoretical predictions for this experimental situation were made based on the general approach of [1], but their correspondence with experiment was only partial. While in [3] both modes are probably excited at the same time, the arguments above show, that the probability of coherent rotation would be increased if the angle between injected spins and magnetization was large. If one believes that coherent rotation can ever be a dominant process, he should first of all search for it in a noncollinear setup.

\section{Field-induced noncollinearity: theoretical predictions}

Here we propose a relatively easy modification of experiment [3] in which the spin-polarization of incoming current is no longer collinear with magnetization and recalculate the switching behavior of the device. Noncolliniarity is created by simply applying external magnetic field in a different direction, as explained on Fig.2. We calculate the "switching diagram" of the device, i.e. find the regions in the $H-j$ parameter space where particular configurations are stable (see [5]). The boundaries between the regions of stability show where current induced switching will happen. A representative "switching diagram" is shown on Fig.3.

If coherent rotation is at least sometimes a dominant process in current induced switching, experiment with noncollinear magnetizations suggested on Fig.2 must give a better agreement with theory in the form $[1,5]$. A positive result of such test would be an important
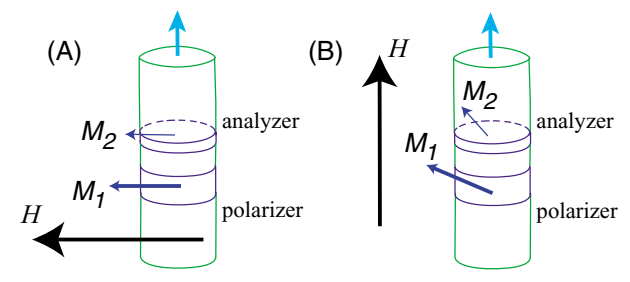

Fig. 2. Cornell nanopillar device [3] in external magnetic field H. A: in original setup with horizontal $\mathbf{H}$ magnetizations $\mathbf{M}_{\mathbf{1}, \mathbf{2}}$ are collinear. B: when $\mathbf{H}$ is applied vertically, an angle between $\mathbf{M}_{1}$ and $\mathbf{M}_{2}$ is created due to different anisotropies of magnetic pieces.

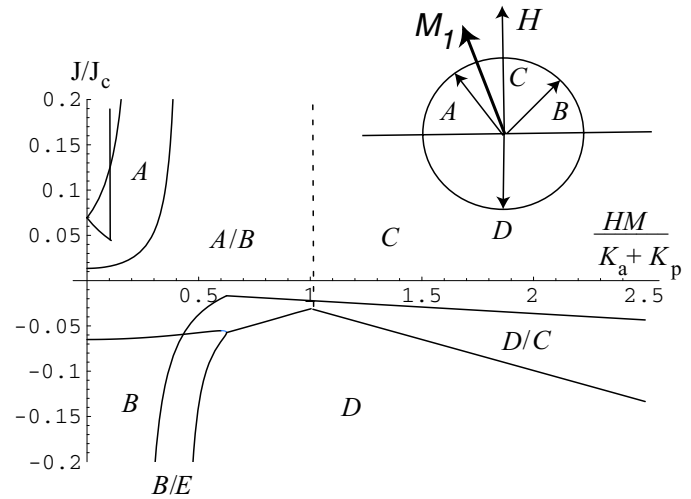

Fig. 3. "Switching diagram" of the nanopillar in a vertical magnetic field. The letters A,B,C, and D on the inset show possible equilibrium directions of $M_{2}$. Same letters on the diagram show the stability region of a particular equilibrium. Two letters signify a bistable region. The analyzer magnet is characterized by horizontal easy plane anisotropy $K_{p}$ and an easy axis anisotropy $K_{a}$ laying in the plane. Their ratio is set to $K_{a} / K_{p}=9$. Current is given in units of $J_{c}=(2 e / \hbar)\left(K_{a}+K_{p}\right) \approx 10^{8} \div 10^{9} \mathrm{~A} / \mathrm{cm}^{2}$. Gilbert damping is set to $\alpha=0.01$.

step in reconciling conflicting points of view on magnetization rotation vs. spin wave generation.

\section{Acknowledgements}

Both authors a grateful to Aspen Center of Physics for hospitality. Y.B. acknowledges the support of U.S. Dept. of Energy, Office of Science, under Contract No. W-31-109-ENG-38, B.A.J. acknowledges ...

\section{References}

[1] J.Slonczewski, J.Magn.Magn.Mater. 159(1996), L1

[2] L.Berger, PRB 33(1986), 1572; PRB 54 (1996), 9353; J.Appl.Phys. 81 (1997), 4880 (1997)

[3] E.B.Myers et al., Science 285 (1999), 867; J.A.Katine et al., PRL 84 (2000, 3149) F.J. Albert et al., Appl.Phys.Lett. 77 (200) 3809;

[4] M.Tsoi et al., PRL 80 (1998),4281; Nature, 406 (2000) 406; J.-E. Wegrowe et al., Europhys.Lett. 56 (2001) 748; Appl.Phys.Lett. 80 (2002) 3361; J.Appl.Phys. 91 (2002) 1; J. Grollier et al., Appl.Phys.Lett. 78 (2001) 3663

[5] J.Z. Sun, PRB 62 (2000) 570; Ya.B. Bazaliy et al., condmat/0009034

[6] J. Miltat et al. J.Appl.Phys. 89 (2001) 6982; J. Slonczewski, J.Magn.Magn.Mater 195 (1999) 261

[7] M.Tsoi, V.Tsoi, JETP Letters 73 (2001), 104; C.Heide et al., PRB 63, 064424 (2001); PRL 87 (2001) 197201 